\title{
Transplante renal de donante vivo: un análisis crítico de estrategias quirúrgicas basado en 40 años de experiencia
}

\author{
Vela Navarrete R, Rodríguez Miñón Cifuentes JL, Calahorra Fernández J, González Enguita C, \\ Cabrera J, García Cardoso JV, Castillon Vela I, Plaza JJ. \\ Departamento de Urología y Unidad de Trasplante Renal. Servicio de Nefrología. Fundación Jiménez Díaz. \\ Universidad Autónoma de Madrid.
}

Actas Urol Esp. 2008;32(10):989-994

\section{RESUMEN}

TRANSPLANTE RENAL DE DONANTE VIVO: UN ANÁLISIS CRÍTICO DE ESTRATEGIAS QUIRÚRGICAS BASADO EN 40 AÑOS DE EXPERIENCIA

Introducción: La prioridad inexcusable de un programa de trasplante renal de donante vivo (TRDV) es la seguridad vital del donante, seguida de la preservación del órgano, obtenido en las mejores condiciones anatómicas y funcionales. La reducción de la morbilidad del donante, inmediata y a largo término, debe ser otro objetivo. Tanto en el TRDV como en el trasplante de cadáver el implante del órgano debe realizarse con las mayores garantías técnicas. Analizamos nuestra mortalidad, morbilidad, incidencias, técnica de la nefrectomía y otros datos relevantes observados en nuestro programa de TRDV iniciado en 1968, con la intención de comparar nuestros resultados con los publicados utilizando técnicas laparoscópicas de nefrectomía, y en consecuencia decidir si nuestro programa debe modificar, o no, sus estrategias quirúrgicas actuales.

Material y métodos: Nuestro programa de trasplante renal se inició en 1964, con riñón de cadáver, y en 1968 con riñón del donante vivo. Desde junio de 1968 hasta junio del 2008 hemos realizado 243 TRDV; un reducido número de trasplantes por año. La técnica de nefrectomía empleada desde 1968-1972 fue el abordaje supracostal propuesto por Turner-Warrick y desde 1972 una miniincisión subcostal, anterior extraperitoneal. La lumbotomía convencional con resección de la última costilla nunca ha sido practicada. El riñón derecho fue utilizado en el $78 \%$ y el implante en la fosa ilíaca derecha en el $85 \%$ La cirugía del donante y el receptor se realizan simultáneamente en dos quirófanos adyacentes, por dos equipos distintos, siendo un solo cirujano responsable de ambos procedimientos; la nefrectomía y el implante. Las complicaciones peroperatorias y postoperatorias, potencialmente relacionadas con la nefrectomía, o de otra naturaleza, han sido evaluadas por los equipos urológicos y nefrológicos.

Resultados: No se han detectado en nuestra serie ningún caso de muerte del donante, pérdida del órgano o complicaciones mayores tales como neumotórax, hemorragias necesitando transfusión, infecciones graves, reintervenciones. Complicaciones menores tales como paresia intestinal, fiebre, infección de la herida operatoria, fueron más frecuentes con el abordaje supracostal que con el miniincisional. Igualmente, este abordaje ha reducido el dolor postoperatorio, consumo de analgésicos y tiempo de recuperación. En el seguimiento a largo plazo la incidencia de hernias incisionales, o paresia de la pared abdominal, no han sido detectadas. La responsabilidad personal de un cirujano en ambos procedimientos soslaya reclamaciones sobre la calidad anatómica y funcional del órgano, facilita la elección de vasos para la anastomosis y reduce el tiempo operatorio y de ocupación de quirófano.

Conclusiones: $\mathrm{El}$ análisis de nuestros resultados cuestiona la conveniencia de sustituir nuestra estrategia quirúrgica actual por los potenciales beneficios e inconvenientes de la nefrectomía laparoscópica, especialmente en un programa de TRDV de reducido número anual. Nuestra técnica de nefrectomía ha ofrecido, a lo largo de una experiencia de 40 años, máximas garantías vitales para el donante y de calidad anatómica y funcional para el órgano, con morbilidad postoperatoria similar a la conseguida con la incisión final laparoscópica.

Palabras clave: Transplante renal. Donante vivo. Nefrectomía. Estrategia quirúrgica.

\section{ABSTRACT}

RENAL TRANSPLANTATION WITH LIVING DONORS. A CRITICAL ANALYSIS OF SURGICAL PROCEDURES BASED ON 40 YEARS OF EXPERIENCE

Introduction: Absolute priority in an LDKT programme are donnor safety and kidney optimal anatomical and functional preservation. Reduced donnor morbidities,both at short and long term,are important objectives. Excellent technical grafting is a must as are the strategies employed for facilitatig it. We revised the incidences of our whole LDKT programme (40 years 243 donors) to confirm if these exigences have been acomplished or a change to new surgical procedures is recommended.

Material and methods: Between 1968-2008 243 nephrectomies and grafting has been performed,a reduced number per year (A cadaver programme has been running simultaneously since 1964). For the nephrectomies a Turner-Warrick apprach was inititialy used and since 1973 a miniincisional, anterior, extraperitoneal approach of approximately $10 \mathrm{~cm}$ in length. The right kidney was removed in $75 \%$ of the cases and the right iliac area for the implant in $85 \%$ In adjacent opperating rooms, one team performs the nephrectomy while the other prepares and dissects free the grafting vessels. Most of the time the same senior surgeon performed both operatios: the nephrectomy and the implant. Peroperative and postoperative complications were evaluated by urologists and nephrologists in charge.

Results: No donors dead,organs lost or major complications in the donors have been documented Minor complications such as intestinal paresia,wound infection,persistent incisional pain were common. Miniincisional abdominal approach reduced postoperative pain and hospital stay (4 days). At long term no incisional hernia or abdominal paresia have been documented. Simultaneous work reduces ischemia time (30-45 s warm: 30 $45 \mathrm{~min}$ cold) and opperatig room occupation(patient preparation plus anesthesia plus operation)estimated in 90-120 min for the nephrectomy and 120-160 for the grafting. The responsibility of the senior surgeon in both procedures facilitates vessel selection for the grafting.

Conclusions: No reasons have been found to reconvert our current nephrectomy procedure to laparoscpic or modify current surgical strategy. Superior safety of open surgery for donors and organs is confirmed. Pain and recovery time are reduced in laparoscopic surgery but not as much when compared with miniincisional approach. Open surgery permits optimal anatomical and functional organ extration facilitatig the quality of the implant. As numbers matter in laparoscopic surgery open nephrectomy isrecommended for reduced LDKT programmes.

Keywords: Renal transplantation. Living donors. Nephrectomy. Surgical strategies. 
$\mathrm{E}^{\prime}$ l éxito y amplia difusión de las técnicas laparoscópicas para la nefrectomía del donante vivo, obliga, a aquellos equipos que llevan años practicando nefrectomías abiertas, a una reconsideración sobre la vigencia de estos procedimientos y la necesidad de incorporar esta nueva técnica en su programa. En centros en los que el programa de TRDV es reducido, la posibilidad de un estudio comparativo es irrealizable. Por otro lado, la nefrectomía abierta tiene muchas variantes, así como la cirugía laparoscópica, y sólo la experiencia de un centro concreto sería válida para comparar beneficios e inconvenientes de ambos procedimientos. La literatura actual sobre la cirugía laparoscópica del donante vivo claramente demuestra beneficios cosméticos, reducción del consumo de analgésico y rápida recuperación del donante, además de un formidable impacto mediático ${ }^{1-3}$. No obstante, la cirugía laparoscópica conlleva singularidades que deben ser valoradas con rigor: tiene una parte de cirugía abierta, la incisión necesaria para la extracción del órgano; implica dificultades técnicas sólo superables en programas numéricamente importantes, con una larga curva de aprendizaje ${ }^{4}$; genera complicaciones específicas de la técnica ${ }^{5-10}$; no se han divulgado, con el rigor necesario, la incidencia de sus complicaciones y en especial de aquellas que son inaceptables en un programa de TRDV, como son la pérdida del donante o del órgano ${ }^{11}$. Igualmente, tampoco se insiste en las potenciales dificultades que la extracción laparoscópica motiva para el ulterior implante del órgano, pero la frecuente extracción del riñón izquierdo, por su vena más larga, explícitamente advierte que existen otras dificultades o inconvenientes para la óptima oferta anatómica del órgano. En consecuencia, es una exigencia ética analizar las referencias prioritarias de un programa de TRDV mantenido durante largos años, antes de embarcarse en procedimientos novedosos y atractivos, incluso calificados como inexcusables ${ }^{12}$. Hemos elegido como referencias básicas de esta comparación, y examinado con rigor en nuestra dilatada experiencia de 40 años, las siguientes: mortalidad y morbilidad del donante; pérdidas de órganos; calidad anatómica y funcional del órgano tras la extracción (decapsulación, dislaceraciones vasculares, calidad y longitud de los vasos, tiempo de isquémica). Incidencia de nuestra estrategia quirúrgica sobre facilidades técnicas para el injerto renal y ocupación de quirófano,. Todo ello ha sido comparado con las más recientes informaciones publicadas por los grupos que de manera mayoritaria practican la nefrectomía laparoscópica ${ }^{13-15}$.

\section{MATERIAL Y MÉTODOS}

Nuestro programa de trasplante renal se inició en 1964, con riñón de cadáver, y en 1968 con riñón del donante vivo. Desde junio de 1968 hasta junio del 2008 hemos realizado 243 TRDV; un reducido número de trasplantes por año. La técnica de nefrectomía empleada desde 1968-1972 fue el abordaje supracostal propuesto por Turner-Warrick y desde 1972 una miniincisión subcostal, anterior extraperitoneal. Las peculiaridades técnicas de esta nefrectomía miniincisional pueden verse en la sección de videos de TTMED Urology (ttmed.com/urology/surgicalvideolibrary) ${ }^{16}$. La lumbotomía convencional con resección de la última costilla nunca ha sido practicada. El riñón derecho fue utilizado en el $78 \%$ y el implante en la fosa ilíaca derecha en el $85 \%$ La cirugía del donante y el receptor se realizan simultáneamente en dos quirófanos adyacentes, por dos equipos distintos, siendo un solo cirujano responsable de ambos procedimientos; la nefrectomía y el implante. Las complicaciones peroperatorias y postoperatorias, potencialmente relacionadas con la nefrectomía, o de otra naturaleza, han sido evaluadas por los equipos urológicos y nefrológicos .

\section{DISCUSIÓN}

\section{Variedades de nefrectomias abiertas}

El término de nefrectomía abierta incluye muy variados abordajes. En 1968, en los pocos centros en los que se realizaban trasplantes renales con donante vivo la técnica habitual era la lumbotomía convencional incluyendo resección de la última costilla $^{17-19}$.

Nosotros comenzamos aplicando el abordaje propuesto por Turner-Warrick ${ }^{20-21}$ que tenía dos claros beneficios sobre la técnica anterior; excluye la resección costal y la frecuente lesión del nervio intercostal con la consecuente paresia abdominal. Sin embargo, aún mantenía el riesgo de lesión pleural, con el neumotórax consecuente, y la lesión de la arteria intercostal con riesgo de hematomas cutáneos e incluso más importantes. Pronto comprobamos que si practicamos la incisión más medial, a partir de la punta de la última costilla y la prolongábamos 
de manera horizontal hasta la fascia del recto mayor del abdomen, conseguíamos los mismos beneficios de la técnica supracostal; incluso con más facilidades operatorias ya que las estructuras abdominales son más fácilmente desplazables hacia un lado $\mathrm{u}$ otro, que los límites que impone el espacio intercostal. La longitud de la incisión fue progresivamente reduciéndose hasta los diez centímetros, incluso a dos centímetros menos en varones delgados y mujeres, o algún centímetro más en varones musculosos, obesidad o donantes añosos. En estos últimos el pedículo vascular debe ser tratado con la mayor delicadeza para evitar tensiones y desgarros de la íntima, más frágil que en individuos jóvenes. El lado derecho, por la posición más baja del riñón y su mayor movilidad, especialmente en mujeres, ofrece más facilidades de disección que el lado izquierdo ${ }^{22}$. Es interesante constatar que algunos cirujanos laparoscópicos practican este tipo de incisión para la extracción final del órgano frente a la propuesta más común de la línea media hipogástrica o Pfanestiel. Esta incisión es utilizada también para la cirugía laparoscópica manoasistida ${ }^{23,24}$. Los músculos abdominales son seccionados y en ningún caso hemos intentado cirugía miofiláctica. Algunos ensayos de nefrectomía por vía pararectal, similar a la incisión realizada en el autotrasplante renal no fueron satisfactorios ${ }^{25}$.

\section{Mortalidad y morbilidad de la cirugia miniincisional y laparoscópica}

No hemos tenido ningún caso del mortalidad o morbilidad mayor operatoria en nuestra serie. Se han constatado dos complicaciones graves, médicas, una motivada por reacción alérgica a la ampicilina y otra debida a una inyección excesivamente rápida de protamina, en la época inicial en la que antes del clamplaje vascular el enfermo recibía 1 mg por kg de peso de heparina junto a un diurético osmótico. Consta en nuestra serie un caso de neumotórax y otro de hemorragia necesitando transfusión y reoperación inmediata verosímilmente motivada por incorrecta ligadura de la vena gonadal. Todas estas complicaciones han sido reconocidas en la cirugía abierta y publicadas ${ }^{26}$. Hasta 1991 se habían publicado 11 casos de muerte en donante vivo ${ }^{27-29}$ pero desde entonces ningún nuevo caso ha sido añadido a la lista. Por el contrario, de acuerdo con la más reciente revisión de la literatura ${ }^{11}$, las muertes y complicaciones mayores motivada por la cirugía laparoscópica parecen estar infradocumentadas. Se han publicado 12 casos de muerte y numerosas complicaciones debidas específicamente a la técnica laparoscópica, tales como incorrecto clampaje de los vasos, con hemorragias y reintervenciones abiertas en el inmediato postoperatorio, reconversiones de la cirugía laparoscópica a cirugía abierta, embolismo gaseoso, perforaciones intestinales por lesión térmica, inmediatas ó tardías, trombosis venosas y otras $^{11,26,30}$. La cuantía y gravedad de las complicaciones ha motivado la demanda de un Registro oficial, de los programas de TRDV con el control consecuente y la oportuna y obligada acreditación de aquellos centros que se reincorporen a este tipo de programa ${ }^{11-31}$.

\section{Pérdidas de órgano y calidad anatómica y funcional del mismo}

En las series de cirugía laparoscópica publicadas se han detectado la pérdida de 18 riñones cuya calidad, por roturas parenquimatosas o dislaceraciones vasculares, hacían inviable el injerto ${ }^{11}$. Todas las extracciones de nuestra serie, así como la literatura sobre cirugía abierta, confirman la ausencia de este tipo de complicación. Otros problemas que limitan la calidad anatómica del órgano están relacionados con decapsulaciones, contusiones y hematomas subcapsulares, y la apropiada longitud y calibre de arteria y vena, así como la potencial lesión de la íntima por tensiones o manipulaciones forzadas del órgano durante la extracción. Uno de los argumentos para utilizar más frecuentemente el riñón izquierdo en la cirugía laparoscópica es la cortedad de la vena renal derecha ${ }^{32}$. En la cirugía miniincisional el espacio operatorio permite con toda facilidad la identificación precisa de la unión de la vena renal con la cava y colocar un Satinsky, si preciso, con la sección de la vena en lugar más distal, soslayando este inconveniente. La cortedad de la vena renal derecha no ha sido en nuestra serie nunca un argumento para descartar la nefrectomía derecha; como señalado en el $78 \%$ de los casos se ha utilizado este órgano.

La calidad funcional del órgano está fundamentalmente relacionada con la exquisitez de las manipulaciones necesarias para su extracción, el tiempo de isquemia caliente y fría, y calidad de la perfusión 
previa al implante. Hasta mediados de los ochenta fue común en nuestra práctica, antes de la sección ureteral y clampaje vascular, heparinizar al enfermo ( $1 \mathrm{mg}$ por $\mathrm{kg}$ de peso) y añadir un diurético esmótico. Se comenzaba el procedimiento de extracción por la sección ureteral y no se practicaba el clampaje vascular sucesivo de la arteria y vena hasta comprobar una excelente diuresis por el uréter seccionado. Desde nuestro incidente con la protamina se optó por una mejor hidratación del paciente, sin más. El tiempo de isquemia caliente en nuestra serie es estimado entre $45-75$ segundos y va seguido de la inmediata perfusión del órgano con líquido de Euro-Collin; en un pequeño contenedor que permite el baño del órgano en hipotermia hasta el comienzo del implante. El tiempo de isquemia fría es variable, en función del sincronismo entre ambos equipos, dificultad en la exposición de los vasos iliacos, ya preparados por el otro equipo, y dificultades en las anastomosis vasculares; se estima entre 30-45 minutos. Se sospecha que la función tardía del órgano implantado está directamente relacionada con el tiempo de isquemica caliente, pero en nuestra serie la causa más común de este retraso estuvo motivada por el efecto nefrotóxico de la ciclosporina ${ }^{22}$.

\section{Dolor postoperatorio, consumos de analgésicos y reincorporación laboral}

La cirugía miniincisional subcostal es mucho menos dolorosa que la lumbotomía convencional. En la estrategia anestésica actual el paciente es controlado las primeras 24 horas mediante bomba de dolor, que ciertamente lo reduce, pero incrementa la incidencia de íleo paralítico. La mayoría de nuestros pacientes podrían abandonar el hospital al cuarto día pero la estancia hospitalaria no es un parámetro de referencia válido en nuestra serie porque nuestros donantes, familiares, prefieren estar próximos al transplantado los primeros días. Razonablemente la incisión en la línea media y de menor longitud debe ser menos dolorosa y por lo tanto es perfectamente asumible que éste sea el gran beneficio de la cirugía laparoscópica. Sin embargo, este beneficio, de extraordinario efecto mediático, no es muy superior al conseguido con la cirugía miniincisional y por si sólo no justifica la incorporación de la cirugía laparoscópica a un programa de TRDV.

\section{Efecto de la extracción sobre la facilidad y calidad de las anastomosis vasculares}

Un órgano bien extraído, con preservación integra de sus estructuras anatómicas (cápsula, arteria, vena, uréter), que son bien identificadas y mejor expuestas durante la perfusión fría, obtenido mediante manipulaciones quirúrgicas que eviten tensión de la arteria o desgarros de la íntima, con secciones vasculares limpias, lineales y no irregulares, facilitará la elección de los vasos idóneos para la anastomosis venosa (cava distal, ilíaca común, bien liberada de sus colaterales que permita su aproximación a la vena renal y no lo contrario) y arteria (ilíaca común o hipogástrica), sin redundancia o tensiones y la precisión necesaria para la mayor rapidez y estanqueidad de las suturas. Un órgano extraído con dificultades causantes de decapsulaciones, dislaceraciones vasculares, extrema cortedad de los vasos, no sólo genera dificultades durante el período de suturas sino que prolongará el tiempo operatorio y de isquemia caliente.

\section{Dos equipos en quirófanos próximos y un solo responsable}

Esta estrategia ha tenido en nuestra experiencia diversas ventajas facilitando aspectos técnicos y operativos. Al practicar la disección del pedículo vascular ya advierte el cirujano datos anatómicos que indudablemente van a condicionar la calidad de las anastomosis vasculares; número de los vasos arteriales (rara vez distintos a los mostrados por las técnicas actuales de imagen) y venosos (más común); calibre de los mismos; divisiones próximas o distales al punto de sección; longitud de arteria y vena, con especial referencia a la longitud de la vena renal derecha: y otros datos. La fácil movilidad del órgano que permite la cirugía miniincisional, incluso su exteriorización, si preciso, sin necesidad de tensiones bruscas o mantenidas, facilita la sección de los vasos en la zona más próxima a su origen, soslayando problemas de longitud. Por otro lado permite alejarse del seno renal preservando grasa y vasos de esta zona así como del uréter, incluyendo la vena gonadal, si oportuno. En definitiva el cirujano alcanza el quirófano donde ha de realizar el implante con informaciones anatómicas precisas que le permiten, con toda rapidez, elegir los vasos más adecuados para la anastomosis, seguramente ya bien disecados por 
el segundo equipo, advertido por comentarios e informes previos. Todo ello implica una considerable reducción del tiempo operatorio.

Pero esta estrategia operatoria permite, además, reducir el tiempo de ocupación de quirófanos, frecuentemente muy superior al tiempo operatorio (tiempo de anestesia y preparación del paciente más procedimiento quirúrgico de piel a piel) y sin interferir en el programa quirúrgico general del Servicio. Nuestros tiempos de ocupación de quirófano han sido estimados en 120 minutos para la nefrectomía y en 180 minutos para el implante. De este modo el programa quirúrgico del día puede continuar sin mayores obstáculos, un dato positivo cuando la ocupación de quirófano es valorada tanto desde el punto de vista económico como funcional, con el rigor con que se hace en la actualidad. En la cirugía laparoscópica los datos que tenemos se refieren al tiempo operatorio y no ocupación de quirófano, y son estimados para la nefrectomía, en las mejores manos, superiores a los 180 minutos $^{33}$.

\section{CONCLUSIONES}

El análisis de las complicaciones observadas en 243 TRDV a lo largo de 40 años, confirma que las prioridades fundamentales e inexcusables en un programa de TRDV se han cumplido satisfactoriamente: no ha habido muertes o pérdidas de órganos ni complicaciones mayores motivadas por la nefrectomía. El abordaje miniincisional reduce el dolor y tiempo de recuperación e impacto estético a condiciones similares a las ofertadas por la nefrectomía laparoscópica. La realización simultánea del procedimiento nefrectomía-implante por dos equipos distintos y un sólo responsable facilita la elección de vasos para el implante y reduce el tiempo de ocupación de quirófanos y de personal.

Este análisis comparativo de nuestra estrategia quirúrgica con la nefrectomía laparoscópica no detecta ningún argumento mayor que nos obligue a sustituir el proceder actual por el laparoscópico. Más aún advierte que en programas reducidos, o iniciales, la nefrectomía abierta ofrece más seguridad que la laparoscópica, que debe reservarse para Centros con programas de elevado número de casos por año y confirmado dominio de las técnicas laparoscópicas. Demorar el comienzo del programa de TRDV argumentando que aún se domina la nefrectomía laparoscópica, cuando aplicable la técnica miniincisional, tampoco parece ser excusa valida. Por otro lado el cirujano laparoscopista debe dominar la cirugía renal abierta ya que sus complicaciones operatorias son resueltas, en muchas ocasiones, recurriendo a la reconversión a esta técnica.

\section{REFERENCIAS}

1. Tooher RL, Rao MM, Scott DF, Wall DR, Francis DM, Bridgewater FH, et al. A systematic review of laparoscopic livedonor nephrectomy. Transplantation. 2004;78(3):404-414.

2. Giessing M. Laparoscopic living-donor nephrectomy. Nephrol Dial Transplant. 2004;19 Suppl 4:iv36-40.

3. Simforoosh N, Bassiri A, Ziaee SA, Tabibi A, Salim NS, Pourrezagholi F, et al. Laparoscopic versus open live donor nephrectomy: the first randomized clinical trial. Transplant Proc. 2003;35(7):2553-2554.

4. Vallancien G, Cathelineau X, Baumert H, Doublet JD, Guillonneau B. Complications of transperitoneal laparoscopic surgery in urology: review of 1,311 procedures at a single center. J Urol. 2002;168(1):23-26.

5. Simon SD, Castle EP, Ferrigni RG, Lamm DL, Swanson SK, Novicki DE, et al. Complications of laparoscopic nephrectomy: the Mayo Clinic experience. J Urol. 2004;171(4):1447-1450.

6. Hazebroek EJ, de Bruin RW, Bouvy ND, van Duikeren S, Bonthuis F, Marquet RL, et al. Short-term impact of carbon dioxide, helium, and gasless laparoscopic donor nephrectomy on renal function and histomorphology in donor and recipient. Surg Endosc. 2002;16(2):245-251.

7. Koffron A, Herman C, Gross O, Ferrario M, Kaufman D, Abecassis M, et al. Laparoscopic donor nephrectomy: analysis of donor and recipient outcomes. Transplant Proc. 2001;33(1-2): 1111 .

8. Boghossian T, Henri M, Dubé S, Bendavid Y, Morin M. Laparoscopic nephrectomy donor death due to cerebral gas embolism in a specialized transplant center: risk zero does not exist. Transplantation. 2005;79(2):258-259.

9. Rehman J, Boglia J, Chughtai B, Sukkarieh T, Khan SA, Lewis $\mathrm{R}$, et al. High body mass index in muscular patients and flank position are risk factors for rhabdomyolysis: case report after laparoscopic live-donor nephrectomy. J Endourol. 2006;20(9): 646-650.

10. Mohamed HK, Lin A, Savage SJ, Rajagopalan PR, Baliga PK, Chavin KD. Chavin, Parenchymal transaction of the kidney inflicted by Endocatch bag entrapment during a laparoscopic donor nephrectomy, Am J Transplant. 2006;6(1):232-235.

11. Shokeir AA. Open versus laparoscopic live donor nephrectomy: a focus on the safety of donors and the need for a donor registry. Urology. 2007;178(5):1860-1866.

12. Kok NF, Lind MY, Hansson BM, Pilzecker D, Mertens zur Borg IR, Knipscheer BC, et al. Comparison of laparoscopic and mini incision open donor nephrectomy: single blind, randomised controlled clinical trial. BMJ. 2006;333(7561):221.

13. Brook NR, Harper SJ, Bagul A, Elwell R, Nicholson ML. Laparoscopic donor nephrectomy yields kidney with structure and function equivalent to those retrieved by open surgery, Transplant Proc. 2005;37(2):625-626.

14. Øyen O, Andersen M, Mathisen L, Kvarstein G, Edwin B, Line $\mathrm{PD}$, et al. Laparoscopic versus open living-donor nephrectomy: experiences from a prospective, randomized, single-center study focusing on donor safety. Transplantation. 2005;79(9):1236- 1240 
15. Simforoosh N, Basiri A, Tabibi A, Shakhssalim N, Hosseini Moghaddam SM. Comparision of laparoscopic and open donor nephrectomy: a randomized controlled trail, BJU Int. 2005;95 (6):851-855

16. Vela Navarrete R. Live donor miniincisional Nephrectomy. tmed.com/urology/surgicalvideolibrary.

17. Murray JE, Harrison JH. Surgical management of fifty patients with kidney transplant including eighteen pairs of twins. Amer J. Surgery. 1963;105:205-218.

18. Penn I, Halgrimson CG, Ogden D, Starzl TE. Use of living donors in kidney transplantation in man. Ach Srurg. 1970;101 (2):226-231.

19. Kaufman JJ, Vela Navarrete R. Trasplantes renales. Rev Mex Urol. 1966;25:497.

20. Turner Warrick RT. The supracostal approach to the renal area. Brit J Urol. 1965;37(6):671-672.

21. Vela Navarrete R, Garcia de la Peña E, Alferez Villalobos C. Consideraciones técnicas sobre el donante vivo de riñón. Rev. Clin. Española 1972;12(6):483-486.

22. Vela Navarrete R, García de la Peña E, Rodríguez Miñón JL, Castillón Vela I. El trasplante renal de donante vivo. En el libro: Trasplante renal; editado por González Martín M. y García Buitrón. Aula Médica. Madrid 2000;121-128.

23. Gershbein AB, Fuchs GJ. Hand-assisted and conventional laparoscopic live donor nephrectomy: a comparison of two contemporary techniques. J Endourol. 2002;16(7):509-513.

24. Rudich SM, Marcovich R, Magee JC, Punch JD, Campbell DA, Merion RM, et al. Hand-assisted laparoscopic donor nephrectomy: comparable donor/recipient outcomes, costs, and decreased convalescence as compared to open donor nephrectomy. Transplant Proc. 2001;33(1-2):1106-1107.

25. Alferez, C, Kaufman JJ, Vela Navarrete, R. Autotrasplante renal por hipertensión maligna vasculorenal en un niño con riñón único funcionante. Rev Clin Esp. 1967;107.
26. Matas AJ, Bartlett ST, Leichtman AB, Delmonico FL. Morbidity and mortality after living kidney donation, 1999-2001: survey of United States transplant centers. Am J Transplant. 2003; 3(7):830-834.

27. Leary FJ, Deweerd JH. Living donor nephrectomy. J Urol. 1973; 109(6):947-948.

28. Uehling DT, Malek GH, Wear JB. Complications of donor nephrectomy. J Urol. 1974;111(6):745-746.

29. Najarian JS, Chavers BM, McHugh LE, Matas AJ. 20 Years or more of follow-up of living kidney donors. Lancet. 1992;340 (8823):807-810.

30. Vastag B. Living-donor transplants reexamined: experts cite growing concerns about safety of donors. JAMA. 2003;290(2): 181-182.

31. Kok NF, Weimar W, Alwayn IP, Ijzermans JN. The current practice of live donor nephrectomy in Europe. Transplantation. 2006;82(7):892-897.

32. Kay MD, Brook N, Kaushik M, Harper SJ, Bagul A, Nicholson ML. Comparison of right and left laparoscopic live donor nephrectomy. BJU Int. 2006;98(4):843-844.

33. Gill IS, Canes D, Aron M, Haber GP, Goldfarb DA, Flechner S, et al. Single port transumbilical (E=Notes) Donor Nephrectomy. The Journal of Urology. 2008;180(2):637-641.

Correspondencia autor: Dr. R. Vela Navarrete Departamento de Urología y Unidad de Trasplante renal Fundación Jiménez Díaz

Avda. de los Reyes Católicos, 2 - 28040 Madrid

Tel.: 915504800

E-mail autor: rvela@fjd.es

Información artículo: Original - Trasplante renal

Trabajo recibido: septiembre 2008

Trabajo aceptado: octubre 2008 